\title{
EXTRACELLULAR PROTEINS IN FUNGI: A CYTOLOGICAL AND MOLECULAR PERSPECTIVE
}

\author{
JOHN F. PEBERDY
}

Microbiology Division, School of Biological Sciences, University of Nottingham, UK

\begin{abstract}
Protein secretion is a vital process in fungi. For many, the secretion of hydrolytic enzymes provides a crucial step in their nutrition in nature. However, in recent years the list of different types of secreted proteins that have been discovered has extended significantly. These have been shown to have a diversity of functions including toxic molecule transport and control of desiccation. The majority of secreted proteins are glycosylated and our understanding of this aspect of fungal biochemistry has also extended in recent years. This review addresses the process of protein secretion from the cytological, biochemical and genetical standpoints. Advances in technology in many areas of scientific approach have enabled a better and understanding of this important process in fungi.
\end{abstract}

Most, if not all filamentous fungi actively secrete proteins during vegetative growth. These molecules are diverse in their function, they include enzymes which play a key role in nutrition and proteins associated with the cell wall, where they may have some structural or recognition role or a protective function as in the case of the recently described hydrophobins, or in the plasma membrane.

The secretion of enzymes is related to the invasive growth that occurs in both saprotrophic and biotrophic filamentous fungi. The former play a key role in the breakdown of plant wastes especially cellulose and lignocellose and many fungi are known to produce the enzymes which hydrolyze these polysaccharides. Both facultative and obligate biotrophs are found associated with plant hosts, and the former also with insects, crustaceans and nematodes. Not surprisingly the plant biotrophs also produce cellulases as well as pectinases, whereas the insect and nematode pathogens produce proteases, lipases and chitinases. In laboratory cultures secreted enzymes are normally detected in the culture medium, however, whether this is true in the natural environment is unknown.

Several enzymes secreted by fungi have realized commercial potential and have application in a variety of industrial processes. Strain development in the fungi used for these purposes has resulted in the production of hyper-secreting strains, which produce yields reportedly as high as $40 \mathrm{gl}^{-1}$ of extracellular protein [1]. This characteristic has highlighted a further application in the production of recombinant proteins [2]. To date 
only two such products have been commercialized, bovine chymosin [3] and lactoferrin [4], however, the yields produced in industrial fermentation are of the order of 1.5 to $2 \mathrm{~g}$, which represents at best only $10 \%$ of the high production of a native protein.

The roles and importance of other types of secreted proteins described above have come to a more detailed understanding in recent times and will, along with a discussion on the processing of proteins for secretion, form the basis of the review that follows

\section{Cell biology of protein secretion}

The first definitive studies on the ultrastructure of the fungal hypha, which aided our understanding of the secretory pathway in filamentous fungi, were published some two decades ago [5]. With the exception of Golgi structures, organelles involved in the post-translational processing of proteins, prior to secretion, present in mammalian cells were also demonstrated in hypha although their spatial distribution was quite different and related to the growth associated polarized organisation which these cells exhibit. The presence of Golgi bodies in filamentous fungi is a matter of debate. Most studies based on transmission electron microscopy have not shown the presence of stacks of Golgi cisternae, but rather single cisternae that have been regarded as Golgi equivalents. This divergence from the typical eukaryotic organisation is further exemplified in studies with the inhibitor Brefeldin A, which was found to cause gross distortion of the endomembrane system at concentrations which inhibit hyphal growth [6, 7]. In contrast ultrastructural examinations on appresoria of fungal pathogens show the presence of this organelle [8]. It is possible that this difference relates to the differentiation of the hyphal tip for the infection step.

The Spitzenkorper is a structure first identified at the tip of the fungal hypha by light microscopy and subsequently shown in transmission electron microscopy to be a complex of apical vesicles [5]. The location of vesicles at hyphal tips suggested a highly polarized release of proteins at growing hyphal apices. Clearly these techniques reveal nothing of the dynamics of such a structure, however, recent studies in which videomicroscopy was used showed the Spitzenkorper to be a "pleomorphic complex constantly changing size and shape" [9]. A co-ordinated role for this vesicle complex in the polarized processes of hyphal tip growth and protein secretion is a reasonable hypothesis that explains the invasive growth characteristic of filamentous fungi. Evidence to confirm this view was made available in studies, which involved the immunostaining of hyphal tips of Aspergillus niger that were secreting glucoamylase [10].

The mechanism of vesicle translocation to the hyphal apex still requires definitive investigation. A reasonable organelle for involvement is the cytoskeleton. Studies involving the use of inhibitors such as methyl benzimidazole-2-yl carbamate suggest a role for both microtubules [11-13] and actin [13], but genetic studies to confirm this are still required. Such data does, however, come from an alternative approach with a videomicroscopic study on a kinesin-deficient strain of Neurospora crassa, which exhibits extensive distortion to hyphal morphology and concomitant failure to assemble a Spitzenkorper [14]. Assuming that kinesin in Neurospora has the same property of 
organelle translocation along microtubules as in mammalian cells, then this data supports the hypothesis of cytoskeleton mediated vesicle translocation.

The role of the secretory pathway organelles has been extensively described in Saccharomyces cerevisiae through studies of conditional mutants defective in secretion and post-translational modification [15]. Studies now being pursued in Aspergillus niger and Trichoderma viride, which will enable a similar confirmation of the functional pathway in filamentous fungi. These investigations will undoubtedly be important in the future with the need to manipulate protein secretion and enhance the process.

\section{Role of the cell wall in protein secretion}

The hyphal cell wall is clearly a porous structure allowing the free flow of small molecules into and out of the cell, however, even the smallest secreted proteins e.g. hydrophobins, are too large to move out of the cell on a simple diffusion gradient. The accumulation of vesicles at the hyphal tips suggests that protein molecules, which have been processed during their passage from the ER to the tip vesicles, will be discharged at the apex as the vesicles fuse to grow the plasma membrane. The apical tip is also the site of nascent wall synthesis, but in this region it is assumed that the different polymers of the wall, i.e. chitin and $\beta$-glucan are independent layers. In the sub-apical region the polymers become cross-linked by covalent and non-covalent bonding to create a rigid wall. The hyphal tips are therefore very plastic and it can be supposed that proteins released on the plasma membrane surface are carried in the flow of cell wall material eventually reaching the outer surface of the wall, where they either remain or are released into the surrounding medium [16].

This model is supported by a variety of studies, which focus on the hyphal wall. Treatment of fungal hyphae with enzyme cocktails used in the isolation of protoplasts confirms the view that proteins are trapped in the more rigid regions of the wall [17]. Studies on the secretion of enzymes by protoplasts demonstrate clearly that without a wall more than $80 \%$ of a secreted protein is found in the external medium [17]. Mutants which affect hyphal morphology and also show a modified wall composition as part of their phenotype may either secrete less or more than the wild type [18] and some cellulose hyper-secreting mutants of $T$. reesei have been shown to have a modified cell wall [19].

\section{Secreted proteins in the plasma membrane and cell wall of fungi}

Since the earliest reports on cell wall composition it has been known that the hyphal wall of fungi contains proteins, however, the chemical format of these molecules and their functional significance were not known. More recent studies have benefited from the technological developments in the past decade and have shown that many of the proteins are glycosylated molecules. Some of these are enzymes destined for secretion, but become entrapped, others are part of the cell wall itself and generally are found at the 
hyphal surface. In the last decade a new type of proteins which have unique properties, called hydrophobins, have been discovered.

The demonstration of the entrapment of enzymes has been described above, however, it is not unlikely that some enzymes are integral parts of the wall and are retained there by covalent or other types of linkages. Such enzymes could be significant in the invasive growth of fungi. Because enzymes can be found free, for example in culture filtrates of growing fungi, it is assumed that this is the norm for the breakdown of extracellular insoluble substrates. Recent studies with Aspergillus fumigatus would tend to suggest otherwise. This pathogen although commonly associated with infections of the lung can be very invasive into deeper body tissues in immunocompromised patients. As the fungus is known to produce a number of proteinases it was reasonably assumed that these were important in tissue invasion. Mutants blocked in the production of these enzymes were, however, found to be still invasive. Analysis of these strains revealed the presence of aspartic and serine proteases in the wall at hyphal tips that could provide a localized mechanism for tissue invasion [20].

Glycoproteins at the cell surface are thought to have a role in recognition or as elicitors. Such proteins are now being discovered in fungi, which have some form of association with plants. One such example is the pathogen of tobacco, Phytopthora parasitica var. nicotianae in which a $34 \mathrm{kDa}$ glycoprotein has been found and purified [21]. This molecule was shown to be an elicitor when tobacco plants exposed to the molecule through their roots showed an up-regulation of lipoxygenase activity and the accumulation of a hydroxyproline-rich glycoprotein. The timing of expression of this glycoprotein was not determined, however, in Colletotrichum lindethianum a proline rich protein, with two putative glycosylation sites, has been found which is expressed in the fungus only when in the host plant [22]. It is suggested that the molecule forms crosslinking components in the cell wall.

Cell wall proteins with recognition functions have been described in ectomycorrhizal fungi in studies on the symbiosis of Psilothus tinctorius and Eucalyptus spp. [23]. Dramatic changes in the cell wall proteins of the fungus were seen at the early stages of the interaction, which implied significant spatial and temporal phenomena. Analysis of the protein profile revealed an increase in a $32 \mathrm{kDa}$ acidic polypeptide and a decrease in a $95 \mathrm{kDa}$ glycoprotein.

The discovery of the unique group of proteins, the hydrophobins is one of the major advances in fungal biochemistry in this decade. Since their discovery in 1991 [24] there have been reports for their presence in many fungi [25] which implies they are probably ubiquitous in terrestrial species. These molecules are generally small peptides comprised of ca 75-90 amino acids, always including 8 cysteine residues, which contribute to the hydrophobic properties. When released onto the surface of aerial hyphae, fruit bodies and other structures exposed to the atmosphere, the proteins self-assemble to form rodlet structures. It is also possible that hydrophobins have a role in adherence of hyphae to surfaces as they grow over them [26] and are involved in plant fungus interactions both symbiotic [23] and pathogenic [27]. When fungi are grown in submerged liquid culture the hydrophobins are released into the growth medium. Molecular analysis of hydrophobin genes has shown that many fungi produce several of these proteins that may be expressed at different stages of development and have different functions. In 
Schizophyllum commune at least four hydrophobins have been identified, SC1, SC3, SC4 and SC6, and their expression shown to be developmentally regulated. SC3 is, so far, unique in that it is the only hydrophobin expressed in the monokaryon of $S$. commune. Synthesis of the protein is suppressed in dikaryons with compatible B mating genes. Expression of SC1, SC4 and SC6 is associated with the dikaryon and fruit body. In these basidiomycete reproductive structures, hydrophobins are important as surface coverings for the fruit body and lining the air cavities in these structures [28]. Families of hydrophobins have also been reported in Agaricus bisporus [29-31], Trichoderma reesei [32-34] and Coprinus cinereus [35].

The importance of glycoproteins in plasma membranes has arisen from quite divergent work on fungal pathogens of animals and plants. The pathogen of mammals Pneumocystis carinii [36] recently designated as a fungus, and the entomopathogen Entomophaga maimaiga [37] have both been shown to have glycoproteins, of yet unknown significance in their plasma membranes. In both Aspergillus fumigatus and plant pathogens, the plasma membrane is the location for glycoproteins that are homologous to $\mathrm{ABC}$ transporters of mammalian cells that are responsible for the efflux of drugs from cells [38]. In fungi transcription of the genes encoding these proteins is enhanced in the presence of fungicides suggesting a role in multi-fungicide resistance [37].

\section{Extracellular secreted proteins}

Probably the best-known examples of proteins secreted from fungal hyphae are hydrolytic enzymes, those which attack plant polysaccharides such as cellulose, hemicellulose, starch and pectin being the most prevalent. However, enzymes that degrade a range of other biological macromolecules and other organic compounds are also found. These enzymes have a significant function in the nutrition of fungi.

Genes encoding many of these enzymes have been cloned and extensively characterized. Two examples of these, which emphasize the advances and developments of recent years, will be described here. These enzymes also represent two good examples of the commercialization of fungal enzymes.

Trichoderma reesei is well known as a highly cellulolytic fungus [40]. The hydrolysis of this polysaccharide is very complex involving three classes of enzymes, cellobiohydrolase (CBH), endoglucanase (EGL) and $\beta$-glucosidase. Genes encoding all three enzymes have been isolated and extensively characterized and as a consequence small families of $c b h$ and $e g l$ genes have been discovered. When grown on cellulose, the expression levels of these enzymes in $T$. reesei are characteristically unequal with the CBH1 protein amounting to $60 \%$ of the total activity produced and secreted. Glucoamylase is an important enzyme produced by Aspergillus niger [41]. In culture the enzyme is produced and secreted when the fungus is grown on starch, maltodextrin or maltose. As far as is known, there is only one form of the protein, which is encoded by the gene glaA. The two proteins show similarities in both structure and in regulation of their synthesis. Each has three domains, a binding domain, a catalytic domain and a bridging domain. How universal this structure is for secreted enzymes remains unknown. 


\section{Protein processing for secretion}

Proteins destined for secretion are synthesized on ribosomes located on the cytosolic surface of the endoplasmic reticulum. The first twenty or so amino acid residues of the translated protein form the signal peptide sequence which has the key role of ensuring translocation of the nascent protein into the lumen of the ER. This is achieved by the docking of the signal peptide with the signal recognition protein, which traverses the ER membrane and mediates the transfer of the nascent protein through the membrane into the lumen of the ER. The complexity of the recognition protein is apparent from the discovery of several genes being involved in $S$. cerevisiae, but recently a gene encoding a protein component of the recognition particle has been isolated from A. niger [42].

\section{Glycosylation and protein folding}

To ensure the secreted proteins to be properly functional when they achieve their destination, several key changes to the molecules are necessary before they are released across the plasma membrane. These changes are known collectively as post-translational modification, but it is becoming increasingly apparent that two of the processes, N-linked glycosylation and protein folding, are probably closely interactive and co-ordinately regulated.

$\mathrm{N}$-linked glycosylation of nascent proteins probably begins very soon after the putative glycosylation sites appear as the polypeptide grows into the ER lumen. The process is best conceived in two stages, the addition of the core glycan to the protein and the maturation of the glycan. The core glycan is comprised of seven sugar residues, two $\mathrm{N}$-acetylglucosamine (GlcNAc), which provide the peptide link between the oligosaccharide and the polypeptide at an asparagine residue, and five mannose (man).

This core molecule is synthesized on the cytosolic ER surface on a transmembrane carrier dolichol phosphate (dol-PP). Synthesis of the core commences with the addition of the two GlcNAc residues to dol-PP catalyzed by the enzyme UDP Nacetylglucosamine:phosphodolichol N-acetylglucosaminyl-1-phosphotransferase (GPT). This enzyme can be found in a microsomal fraction from Aspergillus niger [43] and is sensitive to the antibiotic, tunicamycin. A gene encoding the enzyme has recently been cloned and is currently under investigation (T. Sørenson and J. F. Peberdy, unpublished data). Addition of the five man residues from a GDP-man carrier, catalyzed by dolichol phosphate mannose:Man ${ }_{5} \mathrm{GlcNAc}_{2}$ PPDol mannosyl transferase, completes the core glycan with two branches, one with a single man and the other with four man. The molecule is then translocated into the ER lumen where its maturation takes place by the addition of four additional man residues, by making two branches of two residues from single residue branch, and three glucose residues to the original three man branch. The mature glycan is now transferred to the nascent protein to generate the asparagine $/ \mathrm{N}$ linked structure, a reaction controlled by oligosaccharide transferase.

Simultaneously the nascent polypeptide interacts with chaperone proteins and enzymes that carry out the folding process. Whilst these have been well described in yeast 
and mammalian cells, reports in filamentous fungi are more recent and arise from the isolation of encoding genes, e.g. for BiP and TIG. BiP is a chaperone, which is soluble in the ER lumen; it is constitutively expressed but expression is enhanced by heat shock and exposure of the cells to tunicamycin [44, 45]. Up-regulation by tunicamycin is interesting in view of the target for the antibiotic. The implication is that impaired glycosylation enhances the need for increased BiP levels in the lumen suggesting a link between the addition of the glycan and protein folding. The role of TIG is less well defined, but again the encoding gene is upregulated when glycosylation is inhibited [46].

Alongside these chaperones several enzymes are also involved in the folding process. The one identified so far in filamentous fungi is protein disulphide isomerase (PDI), the gene for which has been cloned [47]. The oxidative environment of the ER lumen is crucial to the functionality of this enzyme.

Further modification to the glycan chains may take place, the timing of which relative to the folding process is not clear. Additional man residues and galactose residues may be added, or indeed removed by mannosidases, resulting in a high degree of heterogeneity to the glycan chains on a particular protein. However, before the protein is released for secretion it is given a "quality control check" in a process involving further enzymes and chaperones. First the two terminal glucose residues are removed a by glucosidases I and II, the protein is then caught with the remaining glucose binding to a membrane associated chaperone calnexin. Interestingly yeast seems to lack this chaperone, but it is present in the mammalian ER together with calreticulin, a lumenlocated chaperone. Attempts to clone a gene encoding this protein from $A$. niger proved to be unsuccessful (J. Lambert and J. F. Peberdy, unpublished data). Whilst bound to calnexin the protein is subjected to the repair of misfolding and then released by the action of glucosidase II cleaving the terminal glucose from the glycan chain. If in the first place all three glucose residues are removed, or the repair of misfolding is incomplete, another enzyme UDP-glucose:glycoprotein glycosyl transferase (GGT) can add a glucose molecule to the glycan chain to enable binding to the chaperone. Evidence for these functions in filamentous fungi is available from the cloning of genes encoding calnexin and GGT (J. Lambert and J. F. Peberdy, unpublished data) and the detection of GGT activity in microsomal fractions (G. Wallis, F. W. Hemming and J. F. Peberdy, unpublished data). The existence of this quality control system is interesting. It is unknown to what degree misfolded proteins are produced in the secretory pathway, the presence of such a complex means of control would imply that it might be a common occurrence.

The above description indicates the importance of N-linked glycosylation in the processing of proteins prior to secretion. However, in both mammalian and fungal cells a second type, O-linked glycosylation also occurs. In this case the process and glycan structures are much less complex. Five membered mannose chains are synthesized, again with a dol-PP GlcNAc anchor but involving the enzyme dol-PP man synthase, which are bonded to threonine-serine residues in the protein. The importance of O-linked glycosylation for secretion is unknown, but its occurrence in specific domains of secreted proteins e.g. the bridge domain of $T$. reesei $\mathrm{CBHI}$ and glucoamylase of $A$. niger may be significant. 


\section{Concluding remarks}

The discussion pursued in this review makes it abundantly clear that the secretion of proteins is a vital process in fungi. It is not surprising therefore that our understanding of the process of protein secretion in filamentous fungi has expanded significantly in the past decade. This reflects the technological developments in all areas relevant to this research from microscopy to molecular biology. The realization that proteins secreted by fungi have a diversity of roles, much wider than enzymes emphasizes even more the importance of the process and the need for continued study. Up to five years ago our interpretation of the secretion pathway in filamentous fungi drew heavily on the extensive genetic studies with $S$. cerevisiae. Whilst there are similarities, it is apparent there are differences not least in the diversity and functional significance of the proteins themselves. Extensive gaps still remain in our knowledge which is clearly very fundamental to either our further exploitation of fungi or for developing new strategies for controlling them in pathogenic situations. It is vital therefore that research on this complex area of fungal biology continues to be pursued.

\section{REFERENCES}

1. van Brunt,J.: Fungi: the perfect hosts. Biotechnology 4, 1057-1062 (1986).

2. Davies, R.W.: Molecular biology of a high-level recombinant protein production system in Aspergillus. In Leong, S.A., Berka,R.M. (eds): Molecular Industrial Mycology. Marcel Dekker Inc, New York. 1991. pp. 45-82.

3. Dunn-Coleman,N.S., Bloebaum,P., Berka,R.M., Bodie,E., Robinson,N., Armstrong,G., Ward,M., Przetak,M., Carter,G.L., Lacost,R., Wilson,L.J., Kodama,K.H., Baliu,E.F., Bower,B., Lamsa,M., Heinsohn,H.: Commercial levels of chymosin production by Aspergillus. Bio-Technology 9, 976-981 (1991).

4. Ward,P.P., Piddington,C.S., Cunningham,G.A., Zhou,X.D., Wyatt,R.D., Conneely,O.M.: A system for production of commercial quantities of human lactoferrin - a broad-spectrum natural antibiotic. BioTechnology 13, 498-503 (1995).

5. Gooday, G.W.:The hyphal tip. In Smith,J.E. (ed.): Fungal Differentiation - a contemporary synthesis. Marcel Dekker Inc., New York. 1983. pp. 315-356.

6. Bourett,T.M., Howard,R.J.: Brefeldin A induced structural changes in the endomembrane system of a filamentous fungus, Magnaporthe grisea. Protoplasma 190, 151-163 (1996).

7. Satait Jeunemaitre,B., Cole,L., Bourett,T., Howard,R., Hawes,C.: Brefeldin A effects in plant and fungal cells: something new about vesicle trafficking? J Microscopy Oxford 181, 162-177 (1996).

8. Hu,G.G., Rijkenberg,F.H.J.: Ultrastructural studies of the intracellular hyphae and haustorium of Puccinia recondita f. sp. tritici. J Phytopath 146, 39-50 (1998).

9. Lopez Franco,R., Bracker,C.E.: Diversity and dynamics of the Spitzenkorper in growing hyphal tips of higher fungi. Protoplasma 195, 90-111 (1996).

10. Wosten, H.A.B., Moukha,S.M., Sietsma,J.H., Wessels,J.G.H.: Localization of growth and secretion of proteins in Aspergillus niger. J Gen Microbiol 137, 2017-2023 (1991).

11. Jochova,J., Rupes,I., Peberdy,J.F.: Effect of the microtubule inhibitor benomyl on protein secretion in Aspergillus nidulans. Mycol Res 97, 23-27 (1993). 
12. Delucas,J.R., Monistrol,LF., Laborda,F.: Effect of antimicrotubular drugs on the secretion process of extracellular proteins in Aspergillus nidulans. Mycol Res 97, 961-966 (1993).

13. Torralba,S., Raudaskoski,M., Pedregosa,A.M.: Effects of methyl benzimidazole-2-yl carbamate on microtubule and actin cytoskeleton in Aspergillus nidulans. Protoplasma 202, 54-64 (1998).

14. Seiler,S., Nargang,F.E., Steinberg,G., Schliwa,M.: Kinesin is essential for cell morphogenesis and polarised secretion in Neurospora crassa. EMBO J 16, 3025-3034 (1997).

15. Rexach,M., Denfert,C., Wuestehube,L., Schekman,R.: Genes and proteins required for vesicular transport from the endoplasmic-reticulum. Ant van Leeu Int J Gen Mol Microbiol 61, 87-92 (1992).

16. Seitsma,J.H., Wosten,H.A.B., Wessels,J.G.H.: Cell wall growth and protein secretion in fungi. Canadian Journal of Botany 73, S388-S395 (1995).

17. Vainstein,M.H., Peberdy,J.F.: Location of invertase in Aspergillus nidulans: release during hyphal wall digestion and secretion by protoplasts. Mycol Res 95, 1270-1274 (1991).

18. Chen,J-S.: Biochemical and genetic aspects of invertase secretion in Aspergillus nidulans. Ph. D. Thesis, University of Nottingham. 1995.

19. Nevalainen,H., Lavygina,L., Neethkling,D., Packer,N.: The biochemical nature of the cell envelope of a high cellulase secreting mutant differs from that of the Trichoderma reesei. J Biotech 42, 53-59 (1995).

20. Reichard,U.: The role of secretory and structure-associated proteinases of Aspergillus fumigatus in the pathogenesis of invasive aspergillosis. Mycoses 41, 78-82 (1998).

21. Sejalon Delmas,N., Mateos,F.V., Bottin,A., Rickauer,M., Dargent,R., Esquerre Tugaye,M.T.: Purification, elicitor activity and cell wall localisation of a glycoprotein from Phytophthera parasitica var. nicotianae, a fungal pathogen of tobacco. Phytopathology 87, 899-909 (1997).

22. Perfect,S.E., O’Connell,R.J., Green,E.F., Doering Saad,C., Green,J.R.: Expression cloning of a fungal proline-rich glycoprotein specific to biotrophic interface formed in the Colletotrichum-bean infection. Plant J 15, 273-279 (1998).

23. Tagu,D., Martin,F.: Molecular analysis of cell wall proteins expressed during the early steps of ectomycorrhiza development. New Phytol 133, 73-85 (1996).

24. Wessels,J.G.H., DeVries,O.M.H., Asgeirsdottir,S.A., Schuren,F.H.J.: Hydrophobin genes involved in formation of aerial hyphae and fruit bodies in Schizophyllum. Plant Cell 3, 793-799 (1991).

25. Kershaw,M.J., Talbot,N.J.: Hydrophobins and repellents: proteins with fundamental roles in fungal morphogenesis. Fun Genet Biol 23, 18-33 (1998).

26. Wosten,H.A.B., Schuren,F.H.J., Wessels,J.G.H.: Interfacial self-assembly of a hydrophobin into an amphipathic protein membrane mediates fungal attachment to hydrophobic surfaces. EMBO Journal 13, 5848-5854 (1994)

27. Talbot,N.J., Kershaw,M.J., Wakley,G.E., de Vries,O.M.H., Wessels,J.G.H., Hamer,J.E.: MPG1 encodes a fungal hydrophobin involved in surface interactions during infection-related development of Magnaporthe grisea. Plant Cell 8, 985-989 (1996).

28. Wessels,J.G.H., Asglerdottir,S.A., Birkenhkamp,K.U., deVries,O.M.H., Lugones,L.G., Scheer,J.M.J., Schuren,F.H.J., Schuurs, T.A., van Weeter,M.A., Wosten,H.B.: Genetic regulation of emergent growth in Schizophyllum commune. Canadian Journal of Botany 73, S273-S281 (1995).

29. DeGroot,P.W.J., Schaap,P.J., Sonnenberg,A.S.M., Visser,J.: The Agaricus bisporus hypA gene encodes a hydrophobin and specifically accumulates in peel tissue of mushroom caps during fruit body development. $\mathrm{J}$ Mol Biol 257, 1008-1018 (1996).

30. Lugones,L.G., Bosscher,J.S., Scholtmeyer,K., de Vries,O.M.H., Wessels,J.G.H.: An abundant hydrophobin (ABH1) forms hydrophobic rodlet layers in Agaricus bisporus fruiting bodies. Microbiology-UK 142, 1321-1329 (1996).

31. Lugones,L.G., Wosten,H.A.B., Wessels,J.G.H.: A hydrophobin (ABH3) specifically secreted by vegetatively growing hyphae of Agaricus bisporus (common white button mushroom). Microbiology-UK 144, 2345-2353 (1998). 
32. Nakari Setala,T., Aro,N., Kalkkinen,N., Alatalo,E., Penntilä,M.: Genetic and biochemical characterisation of the Trichoderma reesei hydrophobin HFB1. Eur J Biochem 235, 248-255 (1996).

33. Munoz,G., Nakari Setala,T., Agosin,E., Penntilä,M.: Hydrophobin gene srh1, expressed during sporulation of the biocontrol agent Trichoderma harzianum. Curr Genet 32, 225-230 (1997).

34. Nakari Setala,T., Aro,N., Ilmen,M., Munoz,G., Kalkkinen,N., Penntilä,M.: Differential expression of the vegetative and spore-bound hydrophobins of Trichoderma reesei - cloning and characterisation of the $h f b 2$ gene. Eur J Biochem 248, 415-423 (1997).

35. Asgeirsdottir,S.A., Halsall,J.R., Casselton,L.A.: Expression of two closely linked hydrophobin gene of Coprinus cinereus is monokaryon specific and down-regulated by the oidl mutation. Fun Genet Biol 22, 54-63 (1997).

36. Vasquez,J., Smulian,A.G., Linkw,M.J., Cushion,M.T.: Antigenic differences associated with genetically distinct Pneumocystis carinii from rats. Inf Immun 64, 290-297 (1996).

37. Bidochka,M.J., Hajek,A.E.: Protoplast plasma membrane glycoproteins in two species of entomophthalean fungi. Mycol Res 100, 1094-1098 (1996).

38. Tobin,M.B., Peery,R.B., Skatrud,P.L.: Genes encoding multiple drug resistance-like proteins in Aspergillus fumigatus and Aspergillus flavus. Gene 200, 11-23 (1997).

39. Del Sorbo,G., Andrade,A.C., van Nistelrooy,J.G.M., van Kan,J.A.L., Balzi,E.E., De Waard,M.A.: Multidrug resistance in Aspergillus nidulans involves novel ATP-binding casette transporters. Mol Gen Genet 254, 417-426 (1997).

40. Tomme,P., van Tilbeurgh,H., Petterson,G., van Damme,J., Vendekerchove,J., Knowles,J., Teeri,T., Claeyssens,M.: Studies of the cellulolytic system of Trichoderma reesei QM9414. Analysis of domain function in two cellobiohydrolases by limited proteolysis. Eur J Biochem 170, 575-581 (1988).

41. Pandey, A.: Glucoamylase research - an overview. Starch 47, 439-445 (1995).

42. Thompson,S.A., Golightly,E.J., Yaver,D.S.: Nucleotide sequence of the Aspergillus niger srpA gene. Gene 167, 337-338 (1995)

43. Wallis,G.L.F., Hemming,F.W., Peberdy,J.F.: Investigation of the glycosyltransferase enzymes involved in the initial stages of the N-linked protein glycosylation pathway in Aspergillus niger. Biochim Biophys Acta 1426, 91-98 (1999).

44. Stedman,T.T., Buck,G.A.: Identification, characterisation and expression of the BiP endoplasmic reticulum resident chaperonins in Pneumocystis carinii. Infection and Immunity 64, 4463-4471 (1996).

45. Van Gemeren,I.A., Punt,P.J., Drint Kuyvenhoven,A., Broekhuijsen,M.P., vant Hoog,A., Beijersbergen,A., Verrips,C.T., van den Hondel,C.A.M.J.J.: The ER chaperone encoding bipA gene of black Aspergilli is induced by heat shock and unfolded proteins. Gene 198, 43-52 (1997).

46. Jeenes,D.J., Pfaller,R., Archer,D.B.: Isolation and characterisation of a novel-stress inducible PDI-family gene from Aspergillus niger. Gene 193, 151-156 (1997).

47. Ngiam,C., Jeenes,D.J., Archer,D.B.: Isolation and characterisation of a gene encoding protein disulphide isomerase, pdiA, from Aspergillus niger. Curr Genet 31, 133-138 (1997). 